\title{
Papel de las empresas consultoras en la gestión del conocimiento
}

\author{
Por Josep M. Rodríguez Rovira
}

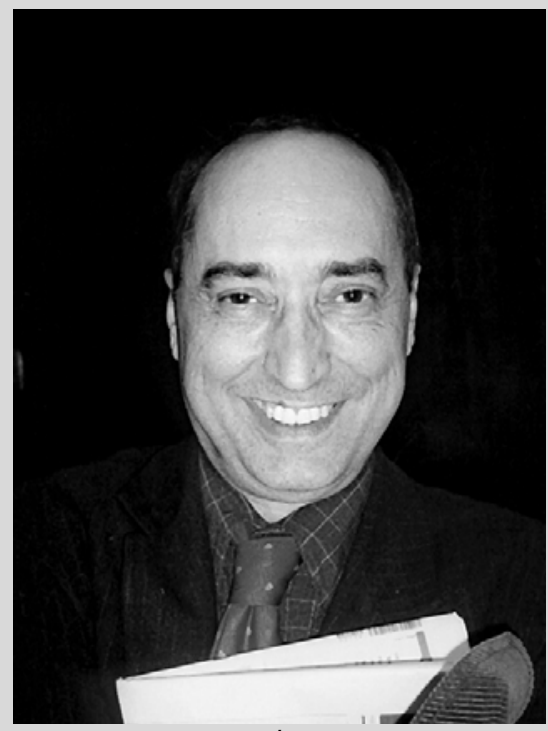

Josep M. Rodríguez Rovira

Resumen: Se ofrece una visión del papel que pueden desarrollar las empresas de consultoría en el ámbito de la organización de sistemas de gestión del conocimiento, haciendo una reflexión sobre algunos criterios a considerar en su implantación. Se realiza también una propuesta de algunos de los servicios que pueden ofrecer a las organizaciones y a los profesionales de la información que trabajan en ellas.

Palabras clave: Consultorías, Gestión del conocimiento, Organizaciones que aprenden, Auditorías de información, Gestión de recursos informativos.

\section{Title: The role of consulting companies in the knowledge management}

Abstract: Consulting companies can play an important role in organisations' successful knowledge management. The article offers an overview of consultancies' activities, including the criteria to be considered when beginning to implement knowledge management processes. Some suggestions are offered regarding the specific services available for organizations and information managers.Consulting companies can play an important role to successful knowledge management in organizations. They offer several aspects that ought to be considered before starting a knowledge management process. The article suggests some specific services that can be offered to organizations and to information managers.

Keywords: Consulting companies, Knowledge management, Learning organizations, Information auditing, Information resources management. Consulting companies, Knowledge management, Learning organizations, Information auditing, Information resources management.

Rodríguez Rovira, Josep M. "Papel de las empresas consultoras en la gestión del conocimiento". En: El profesional de la información, 2000, septiembre, v. 9, n. 10, pp. 11-16.

\section{Qué puede aportar una empresa consultora}

1. A menudo las soluciones vienen de fuera. Así es con frecuencia, las aportaciones de consultores externos son fundamentales para la resolución de problemas o la implantación de nuevos objetivos en las organizaciones. Todo el mundo sabe que la consultoría, en los ámbitos más clásicos de la gestión empresarial, es un servicio cada vez más ampliamente utilizado por las empresas y por todo tipo de organismos, incluidas las administraciones. De ahí el gran éxito que están cosechando.

Entre las actividades de consultoría, las relacionadas con el mundo de la información están adquiriendo una importancia creciente. El boletín SVP news publica unas estadísticas sobre este sector en España en donde se refleja que su volumen de facturación en el año 1998 fue de 194.700 M PTA, con un crecimiento del 19,4\% respecto del año 1997. De los 10 sectores que considera la estadística publicada, el de mayor facturación es el de desarrollo e implantación de sistemas de información, con un total de $85.000 \mathrm{M}$ en el año 1998 y un crecimiento del 22,6\% sobre el anterior. El segundo sector es el de estudios de mercado, con
$37.000 \mathrm{M}$ y un incremento del $25 \%$, siendo el tercero la consultoría en tecnologías de la información, con una facturación en 1998 de 20.000 M y un crecimiento del $22,6 \%$.

«Con la colaboración de una
empresa de consultoría se ob-
tienen conocimientos acumula-
dos, experiencia y trabajo profe-
sional especializado, con los
consiguientes ahorros de inver-
sión y de tiempo, mejorando
con ello la productividad de la
organización»

Vemos pues que de los tres de mayor facturación y desarrollo, dos están relacionados con los sistemas y las tecnologías de la información. El conjunto de estos dos sectores supone el $54,7 \%$ del total de facturación en consultoría en España en 1998.

2. ¿Que aporta una empresa de consultoría? Constituyen un soporte muy útil para las organizacio- 
nes y muy especialmente para los profesionales que trabajan en ellas. Esto es así porque ofrecen:

Una visión global y externa a la organización, no condicionada por su propia dinámica y las circunstancias que la rodean.

Conocimientos de profesionales especializados.

Experiencias acumuladas en ámbitos concretos de actividad.

Incremento de la capacidad de trabajo concentrada en un área y un tiempo determinados.

Como resumen, con la colaboración de una empresa de consultoría se obtienen conocimientos acumulados, experiencia y trabajo profesional especializado, con los consiguientes ahorros de inversión y de tiempo, mejorando de esta forma la productividad de la organización. Además, todo ello permite que los profesionales de la institución concentren su actividad en los temas que son propios de la misma, en el negocio principal, aquel que la organización domina y en el que es realmente competitiva (el llamado core business). Los criterios actuales de gestión gerencial de organizaciones tienden a externalizar las actividades que no forman parte del núcleo básico.

3. Lo que demanda el mercado. Los especialistas coinciden: estamos en los inicios de un cambio de época en la historia humana. El sistema de producción industrial automatizado surgido de la Segunda Guerra Mundial y los modelos económicos, sociales y políticos que le han dado soporte durante los últimos cincuenta años, está agotándose. Asistimos al nacimiento de una nueva época, en la que la información y el conocimiento adquieren categoría de fuerzas de producción directa. Es más, la producción material es cada vez más el resultado del conocimiento y su gestión eficaz. Los fenómenos de la globalización económica están en la base de los objetivos expansivos de este nuevo modelo, así como de los cambios ideológicos y sociales de los que estamos siendo protagonistas.

\section{«Asistimos al nacimiento de una nueva época, en la que la información y el conocimiento adquieren categoría de fuerzas de producción directa»}

En este contexto, el mercado demanda de los profesionales de la información su aportación para contribuir a dotar a las organizaciones de la preparación y los instrumentos que les permitan un uso intensivo de la información y del conocimiento. Y esto como condición que garantice su viabilidad y su competitividad en el futuro.

\section{Una propuesta de criterios}

1. Las organizaciones deben aprender. Dentro de veinte años el arquetipo de gran empresa tendrá la mitad de niveles de dirección y tan sólo un tercio de los ejecutivos que hoy necesita.'La aceleración de los cambios que vivimos es tal que, aunque ha transcurrido poco tiempo desde que Peter F. Drucker hizo esta afirmación, seguramente los veinte años previstos podrían reducirse considerablemente. Cómo será posible que se cumpla la predicción? Pues creando organizaciones capaces de aprender.

Aprender de quién? En primer lugar, y fundamentalmente, de ellas mismas, de los conocimientos y la experiencia de sus propios trabajadores; también de sus clientes, de sus proveedores y de la competencia.

Cómo aprender? Con la decisión corporativa necesaria para crear las condiciones y dotarse de los medios necesarios. Con el compromiso radical de la alta dirección. Con la cooperación activa y el entusiasmo, casi cabría decir, de las personas que trabajan en ella.

Algunas definiciones de organización que aprende realizadas por especialistas en esta materia, nos pueden ayudar a situar este objetivo. Hemos elegido las siguientes:

Ena entidad aprende si, mediante el procesamiento de la información, cambia la gama de sus potenciales conductas." George P. Huber, citado por David A. Garvin.

Se considera que una organización aprende cuando traduce las enseñanzas obtenidas de su pasado en normas que orientarán su conducta." Barbara Levit, citada por David A. Garvin.

Como se puede observar, el denominador común de estas definiciones es el uso de la información para evaluar conductas y sus resultados, así como para deducir de sus enseñanzas comportamientos futuros. Por ello, para aprender son necesarias políticas adecuadas a los objetivos de cada organización, así como los recursos, las metodologías, la definición de procedimientos, los objetivos cuantificables y las métricas de evaluación de resultados.

2. Del documento al conocimiento táito. Una parte fundamental del proceso de aprendizaje de una organización es la obtención de conocimiento de sus empleados. De esta adquisición de conocimiento de los trabajadores hay una parte en la que la organización puede influir muy poco o nada: la experiencia personal y emocional, su capacidad de reflexión y asimilación, su formación académica y su experiencia anterior. Pero sí hay otros elementos en los que la empresa puede intervenir positivamente. De entre ellos se encuentran: formación, políticas de motivación y ges- 
tión de la información y del conocimiento. Nosotros nos referiremos sólo a esta última.

Por lo que respecta a la información, una de las vías básicas de obtención de la misma, tanto la externa a la organización como la interna, es el acceso a documentación, sea cual sea el soporte: papel, información oral, gráfica, vídeo o acceso a bases de datos. A su vez, el proceso de adquisición de conocimientos tiene dos componentes básicos: uno la asimilación de conocimiento de otras personas, el otro, el propio aprendizaje por la experiencia.

Las nuevas tecnologías de la información posibilitan una difusión del conocimiento capaz de incorporar más y más contenidos procedentes de documentos de diversos formatos, y a favorecer un proceso de interiorización y reflexión en donde intervienen cada vez más personas. Es decir, un recorrido que va del documento, como expresión de conocimiento acumulado, al conocimiento tácito de las personas.

3. Del conocimiento táito al explícito. En un proceso constantemente bidireccional no sólo adquirimos y asimilamos conocimiento, sino que también lo exteriorizamos y transmitimos. Esta transferencia podemos realizarla mediante diferentes formas de expresión y soportes, cuanto más sofisticados más complejos y costosos. Compárese la diferencia entre una conversación entre personas y la producción de un vídeo o una película.

Las más sencillas, y por ello las más utilizadas, son las formas verbales y las escritas porque además dan total autonomía al emisor, es decir, al que se expresa, al que transmite conocimiento, que no necesita intermediarios para manifestarse ni medios sofisticados de difusión.

Desarrollar las mejores y más eficientes formas para que el conocimiento se exprese, se convierta de tácito en explícito y sea útil a la organización, es una nueva responsabilidad y un reto para profesionales y empresas mediante técnicas para la realización de auditorías de la información, análisis de circuitos de información, diseño de bases de datos, etc.

4. El aprendizaje eficiente. La ecología del conocimiento. Los recursos de las organizaciones son limitados y es necesaria su economía para obtener re-

Los contenidos de El profesional de la información están protegidos por copyright. Pueden ser reproducidos hasta un máximo de dos por número (total o parcialmente), siempre que se cite la procedencia. sultados altos con inversiones reducidas. ¿En quése parece una empresa a un bosque? A la hora de abordar un proyecto de gestiń del conocimiento vemos que puede beneficiarse de los criterios de acciń ecolǵica de la naturaleza, entre ellos:

La interrelación e interdependencia entre los diferentes componentes de un determinado ambiente. Toda actuación en un ámbito tiene repercusiones en otros.

El entorno continuamente cambiante, lo que exige una atención permanente y capacidad de reacción y de anticipación.

La gestión de recursos, los cuales son limitados, y economizarlos debe ser un objetivo permanente.

La reutilización de los recursos es otra de las medidas imprescindibles, para la naturaleza y para los procesos de información.

Una organización, una empresa, no es una suma de procesos secuenciales y lineales, sino que es como un organismo vivo donde cada acción, sobre todo en el ámbito informativo, tiene un tejido de consecuencias en diferentes áreas de la actividad.

«Una entidad aprende si, mediante el procesamiento de la información, cambia la gama de sus potenciales conductas» (George P. Huber)

Las empresas de consultoría están en las mejores condiciones de proporcionar un importante valor añadido a los procesos de tratamiento de la información, por la aportación que suponen la formación y experiencia del personal que trabaja en las mismas.

5. Visiń integrada e integradora. Una empresa consultora debe trabajar para el cliente pero debe, sobre todo, trabajar con él. Para ello debe conocerlo y poner en práctica una metodología de trabajo y relación que tenga muy en cuenta que abordar con garantías la gestión del conocimiento en una organización requiere un planteamiento capaz de integrar dinámicamente lo que son los elementos definitorios más críticos de toda organización, a saber: misión, visión, planes estratégicos, objetivos, valores corporativos, cultura organizacional, estructura y procesos básicos. Partir de estas premisas es necesario para asegurar que las propuestas que se realicen al cliente serán acordes con sus objetivos.

\section{Propuesta de servicios}

1. Explosiń de oportunidades. Ahora existen para las empresas consultoras y para los profesionales 
de la información multitud de oportunidades de trabajo. Es tan amplio el abanico de posibilidades que parece una situación creada ex profeso para ellos. Se combinan diferentes circunstancias que dan este óptimo resultado:

La gestión del conocimiento no es sólo una moda. Como sucede en el caso de internet, hay una conciencia profunda y generalizada de que esto va en serio y que hay que actuar y posicionarse.

Đe aquí la importancia creciente que reconocen todas las organizaciones al papel de la información y, dentro de ella, a la documentación.

\section{«Desarrollar las mejores y más eficientes formas para que el conocimiento se exprese es una nueva responsabilidad para profesionales y empresas me- diante técnicas para la realiza- ción de auditorías de la informa- ción, análisis de circuitos de in- formación, diseño de bases de datos, etc.»}

Todo ello, se une a fenómenos no menos profundos en el ámbito de las concepciones de gestión empresarial y, de manera destacada, las actuales políticas de relaciones humanas tendentes a la motivación, la integración, la participación activa de los trabajadores...

La evolución de las tecnologías de la información y, especialmente, las asociadas a intranet/extranet/internet.

La creciente capacidad y rapidez de las comunicaciones con la capacidad de interconexión que conllevan: WAP, TV digital/internet.

Por esta razń para muchas organizaciones, y los profesionales que trabajan en ellas, puede ser muy til la aportaciń y soporte de empresas especializadas para llevar a cabo:

procesos de auditoría de la información y de soporte a la gestión del conocimiento,

ebtención de recursos externos de información, y

procesos y actuaciones necesarios para su proyección corporativa tal y como se enumera a continuación:

\section{a. La gestiń del conocimiento corporativo.}

Para el máximo aprovechamiento del conocimiento corporativo se trata de actuar de manera que se consigan dos objetivos. El primero es el acceso intensivo, por parte de los miembros de la organización, a la información que ésta genera y al conocimiento que cada trabajador tiene de su propio trabajo, de su relación con clientes y proveedores en todo aquello que pueda ser útil al resto de compañeros. El segundo es hacerlo con criterios, cauces e instrumentos que aseguren, además de la actualización regular, un proceso de aprendizaje y mejora constante por el colectivo.

Auditorías de la informaciń. Comportan el análisis de los recursos de información/conocimiento que genera la organización y los que obtiene del exterior, así como la definición de objetivos para su uso y aprovechamiento de manera que se aseguren el correcto cumplimiento de la misión y los objetivos corporativos. Algunos de los elementos componentes de las auditorías son:

-determinación de recursos y fuentes de información,

-establecimiento de los procesos básicos y recursos de información asociados,

-definición y diseño de procedimientos para el uso de la información/conocimiento,

planes estratégicos sobre necesidades de información,

-diseño de mapas de gestión del conocimiento,

-diseño de servicios de tratamiento, difusión y gestión de la información,

evaluación de servicios de gestión de la información, y

planes de calidad en el uso de la misma.

Planes estratǵicos para intranets corporativas. Las intranets juegan un papel determinante en la gestión de la información y el conocimiento. Puede decirse que son un componente tecnológico/organizativo clave en todo proyecto. Hablar de intranet significa hablar no sólo de información, sino también de procesos y procedimientos, de relaciones humanas, de calidad, de inteligencia comercial, etc. Por ello, su definición y diseño forman parte del núcleo básico de la actividad corporativa. Los consultores pueden aportar a las organizaciones su contribución en:

planes estratégicos de intranets corporativas,

-diseño de bases de datos específicas,

instalación de programas para su gestión,

tratamiento de imágenes,

gestión electrónica de documentos, e

informatización de bibliotecas. 
Externalizaciń de servicios. La gestión de la información/documentación/conocimiento en las organizaciones es, a la par que cada vez más necesaria, más compleja. Por ello la tendencia a externalizar (subcontratar servicios a una empresa especializada) se generaliza cada vez más. Las empresas consultoras pueden aportar conocimientos, experiencia y tecnología para cubrir las necesidades de las organizaciones en diferentes ámbitos:

centrol integral de departamentos de gestión del conocimiento, centros de documentación y bibliotecas,

análisis y creación de metadatos,

-digitalización y descripción de información en todo tipo de soporte, $\mathrm{y}$

catalogaciones documentales y bibliográficas.

Formaciń a medida. La adecuación del personal, su adaptabilidad a nuevas necesidades y entornos, el trabajo en equipo, la motivación, son componentes esenciales del nuevo paradigma en la gestión de recursos humanos en las organizaciones. Para todo ello es imprescindible la formación continuada, en especial cuando hablamos de tecnologías de la información: luación,

procesos de gestión de la información y su eva-

recursos y tecnologías de la información y la documentación,

gestión y edición electrónica de información y documentos,

calidad en los servicios,

satisfacción del cliente/usuario, y

marketing de los servicios de información.

«Una empresa de consultoría está en las mejores condiciones de proporcionar un importante valor añadido a los procesos de tratamiento de la información, por la aportación que suponen la formación y experiencia del personal que trabaja en estas empresas»

\section{b. La proyecciń corporativa.}

La proyección hacia el exterior (clientes, usuarios, ciudadanos y proveedores) de las organizaciones es una exigencia de política informacional y de la globalización. Entre los muchos recursos para llevarla cabo, los electrónicos juegan un papel crecientemente importante.
Ediciń electrúica. La presencia en internet condiciona ya la estrategia y las políticas de inversión de las organizaciones, tal es su importancia. Los contenidos son el valor más importante. Los profesionales de la información/documentación están entre los que reúnen mejores condiciones para la definición y la elaboración de contenidos en los aspectos básicos de la presencia en internet:

planes estratégicos de portales y webs corporativas,

-definición de pautas y criterios de presencia corporativa,

búsqueda, selección, elaboración e implementación de contenidos,

edición de información profesional para su difusión en cd-rom e internet,

-diseño de aplicaciones para acceso a la información,

tratamiento de la información para su difusión $\mathrm{y}$ acceso en diferentes formatos,

difusión de la información, y

alojamiento de la información en servidores internet.

\section{«La presencia en internet condi- ciona ya la estrategia y las polí- ticas de inversión de las organi- zaciones»}

Buscadores temficos. Tener actualizada, clasificada y accesible la información de las webs de las empresas de la competencia, o un conjunto de páginas cuyo contenido temático nos interesa especialmente, es ahora posible con el uso de buscadores capaces de recorrer con regularidad las direcciones web que nos interesen y de crear bases de datos con la información recogida en ellas:

creación de bases de datos temáticas de recursos en internet,

alojamiento de bases de datos para acceso exclusivo de clientes, e

instalación de programas específicos para búsqueda de información en internet.

Programas de gestiń de servicios en internet. La eficiencia en el uso y difusión de la información requiere de herramientas que faciliten la realización de actividades cada vez más frecuentes y necesarias, como:

el envío de documentos por la Red, 
ła gestión estadística de accesos en las webs o intranets corporativas, $\mathrm{y}$

la revisión automática y periódica de direcciones URL.

\section{c. Recursos externos de informaciń.}

Ninguna organización es autosuficiente informativamente sino que, por el contrario, las necesidades de obtención de información externa crecen constantemente. Es por ello que la aportación de consultores especialistas es de una inestimable ayuda para la obtención y tratamiento de información profesional.

\section{«Ninguna organización es auto- suficiente informativamente si- no que, por el contrario, las ne- cesidades de obtención de in- formación externa crecen cons- tantemente»}

Acceso a recursos electróicos. La información profesional, científica, técnica, económica, de mercados, legal, etc., ya se obtiene de manera creciente en formato electrónico y no sólo como bases de datos, sino también en texto completo. Además, cada vez es mayor el proceso de integración de posibilidades de búsqueda y de combinación de contenidos. En muchos casos se puede pasar ya de las referencias obtenidas en una búsqueda en bases de datos al texto completo (con imágenes incluidas) de los artículos referenciados. De las empresas especializadas se pueden obtener servicios de valor añadido de gran utilidad:

-datos sobre recursos de información disponibles en el mercado,

-distribución y suscripción de bases de datos profesionales,

servicios de suscripción y acceso a revistas electrónicas, y

suministro de artículos originales.

Instalaciones para el acceso a la informaciń. Acceder a ella y consultarla de forma eficiente es un objetivo para cuya consecución existen herramientas que lo hacen posible:

servidores intranet/extranet/internet,

sistemas específicos de los editores para acceso a la información, y

sistemas cliente mínimo para corporacionescorporaciones (programas que se ejecutan en el servidor de internet/intranet y que no hace falta tener instalados en el propio ordenador, tipo Metaframe). d. Cooperar con el cliente para que sea líder en su propio mercado. Como colofón, cabe decir que uno de los objetivos básicos de las empresas de consultoría debe ser contribuir eficazmente a que sus clientes sean líderes en su propio mercado, se encuentre éste compuesto de clientes, usuarios o ciudadanos, según estemos hablando de empresas, instituciones diversas o administraciones. Conseguirlo forma parte de su compromiso profesional con los clientes y es la forma de garantizar su continuidad en el futuro. Para ello deben actuar guiadas por unos valores corporativos que les deben ser reconocidos por clientes y círculos profesionales: calidad, innovación, cooperación, confidencialidad y profesionalidad. Es decir, que la actuación de la empresa consultora debe estar guiada por la obtención de óptimos resultados por parte de su cliente en su ámbito de actividad.

\section{Bibliografía}

Bueno, E.; Morcillo, P. La dirección eficiente. Madrid: Pirámide, 1993. Isbn: 84-368-0749-9.

Cowan, Les. Reorganizing organizations and information: how knowledge technologies squash hierachy and alter the role of information.'En: Document image automation, 1991, septiembre-octubre, pp. 288-294.

Drucker, Peter F. Łlega una nueva organización a la empresa.'En: Harvard business review: gestión del conocimiento. Bilbao: Deusto, 2000, pp. 1-22. Isbn: 84-234-1724-7.

Galgano, A. Calidad total. Madrid: Díaz de Santos, 1993. Isbn: 84-7878-094-0.

Garvin, David A. Crear una organización que aprende.'En: Harvard business review: gestión del conocimiento. Bilbao: Deusto, 2000, pp. 51-90. Isbn: 84-234-1724-7.

Knowledge manager: making it work. Consultado en: 25-06-2000. http://www.skyrme.com/pubs/lawlib99.htm

Leading lights: an interview with author and consultant Stan Davis. Consultado en: 25-06-2000.

http://webcom.com/quantera/davis.html

Stan Davis. Leading lights: an interview with author and consultant. Consultado en: 25-06-2000.

http://webcom.com/quantera/davis.html

Mas, J.; Ramió, C. La auditoría operativa en la práctica. Barcelona: Marcombo, 1997. Isbn: 84-267-1123-5.

Reddin, B.; Ryan, D. Manual de dirección por objetivos. Bilbao: Deusto, 1995. Isbn: 0-07-451869-0.

Skyrme, D. The next step.' En: International consultants' guide, 1999, julio, pp. 8-10.

SVP news. Revista bimestral de información, 2000, abril-mayo, n. 17.

Josep M. Rodríguez Rovira, director gerente, DOC6 Consultores en recursos de información.

jmrodriguez@doc6.es 


\section{El acceso a un mundo de información}

En un mundo en el que todo cambia tan de prisa, las cualidades que más se valoran en un socio son visión de futuro, experiencia y fiabilidad. Bien sea gestionando suscripciones o bien suministrando artículos directamente a su ordenador, Swets Blackwell provee servicios de calidad a bibliotecas y centros de información de todo el mundo.

Swets Blackwell aumenta la capacidad del conocimiento

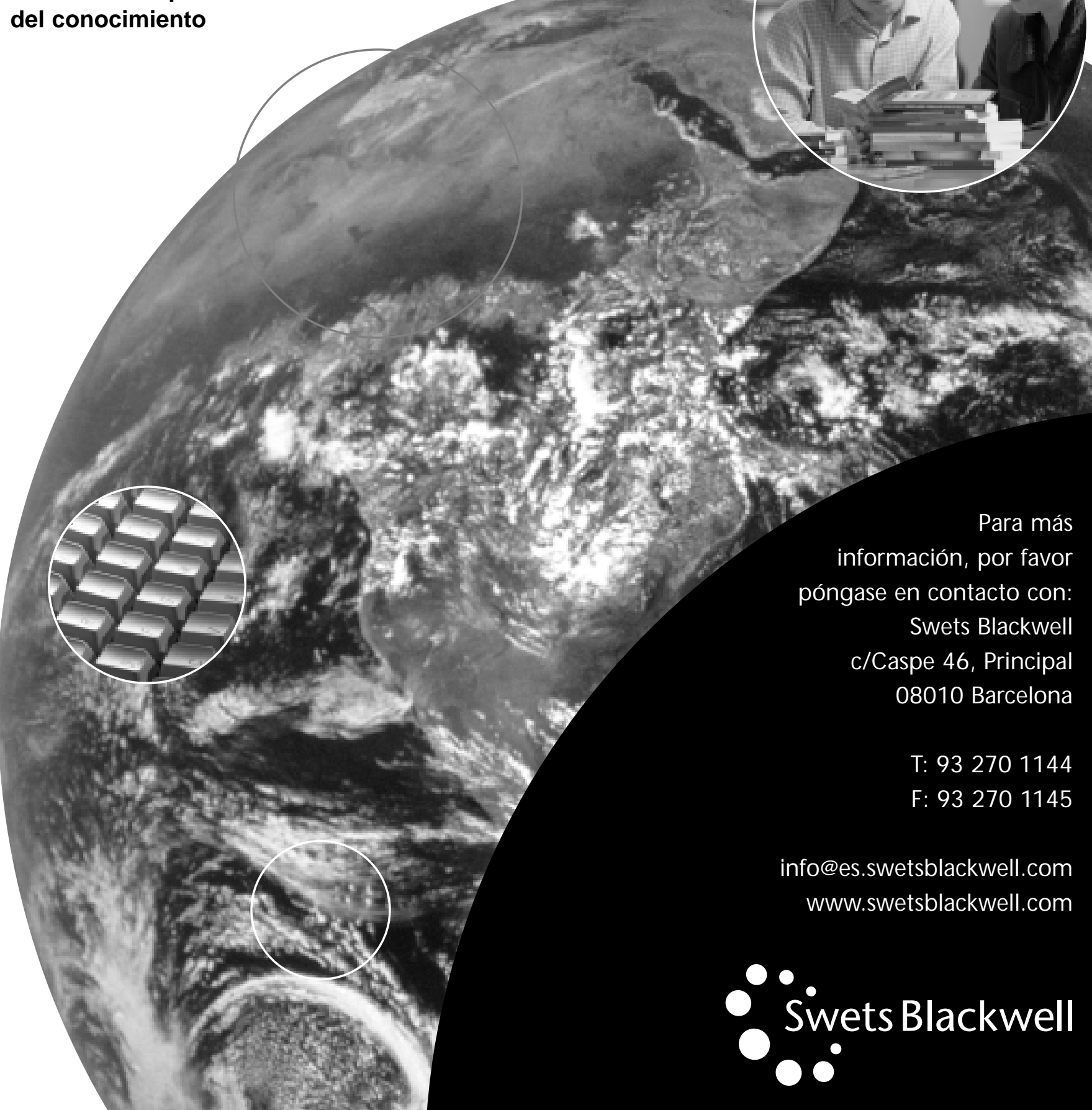

